\title{
PEDAGOGY
}

\section{МЕЖПРЕДМЕТНАЯ СВЯЗЬ - КАК ПУТЬ ПОВЫШЕНИЕ ЭФФЕКТИВНОСТИ ОБУЧЕНИЯ В ПРОЦЕССЕ ПРЕПОДАВАНИЯ КУРСОВ „ОБЩЕЙ ФИЗИКИ И МАТЕМАТИКИ“ В БГМА С ПРИМЕНЕНИЕМ КОМПЬЮТЕРНЫХ ТЕХНОЛОГИЙ}

\author{
Диасамидзе Мзия Ресановна, \\ кандидат физико-математических наук, профессор, департамент базовых дисииплин, \\ факультет навигации, Батумская Государственная Морская Академия, г. Батуми, Грузия \\ Телия Тамаз Николаевич, \\ кандидат технических наук, ассоциированный профессор, департамент базовых дисииплин, \\ факультет навигаџии, Батумская Государственная Морская Академия, г. Батуми, Грузия
}

Моцкобили Ия Ревазовна,

доктора информатики, ассоииированный профессор, департамент базовых дисииплин, факультет навигачии, Батумская Государственная Морская Академия, г. Батуми, Грузия

DOI: https://doi.org/10.31435/rsglobal_wos/31052020/7092

\section{ARTICLE INFO}

Received: 09 March 2020

Accepted: 07 May 2020

Published: 31 May 2020

\section{KEYWORDS}

interdisciplinary communication, learning efficiency,

temperature coefficient of

resistance,

thermistor,

correlation,

approximation.

\begin{abstract}
The article analyzes the influence of the relationship of basic subjects with general technical subjects in the learning process for students of marine specialties. It is possible to realize this connection by developing and implementing engineering problems that a student at the BSMA will have to face both in primary courses and in the study of special subjects. Our early research results indicate the effectiveness of the use of such a teaching method as a means of developing students' thinking. Our institution is a specific technical university. Graduates have to work in extremely dangerous situations at sea, and they have to decide the fate of passengers, ship and cargo in a matter of minutes. Therefore, special thinking is an essential feature of a sailor. While studying, along with other subjects, students study various marine equipment, conduct observations of measuring instruments (for example, temperature in the engine room, in turbine pumps, etc.). The article considers the engineering problem - determining the value of the linear temperature coefficient of a thermistor in Excel. Based on the basic data, a graph of the dependence of temperature on resistance is constructed, a linear temperature coefficient and the reliability of the approximation value are determined. It is established whether the device used is laboratory. The corresponding conclusions are made.
\end{abstract}

Citation: Diasamidze Mzia, Telia Tamazi, Motskobili Ia. (2020) Intersubject Communication - as a Way to Increase the Effectiveness of Training in the Teaching of Courses "General Physics and Mathematics" in the BSMA. International Academy Journal Web of Scholar. 5(47). doi: 10.31435/rsglobal_wos/31052020/7092

Copyright: (C) 2020 Diasamidze Mzia, Telia Tamazi, Motskobili Ia. This is an open-access article distributed under the terms of the Creative Commons Attribution License (CC BY). The use, distribution or reproduction in other forums is permitted, provided the original author(s) or licensor are credited and that the original publication in this journal is cited, in accordance with accepted academic practice. No use, distribution or reproduction is permitted which does not comply with these terms.

Введение. Техническое образование рассматривается, как важнейшая составляющая фундаментальной подготовки бакалавра, т. к. является мощным средством - при решении 
прикладных и инженерных задач, умения логически мыслить, использовать знания для решения профессиональных задач, оперировать с абстрактными объектами и т.д. Факт, что студенты в рамках школьного курса владеют некими основами работы на компьютере, им это интересно, но не имеют достаточного знания Вышей математики и Общей физики. Каждый опытный педагог выбирает и создает новый инструментарий для достижения цели педагогической деятельности повышения эффективности учебного процесса. Целью нашего исследования является теоретическое и практическое обоснование эффективности использования компьютерной технологий в процессе обучения. В ранних публикациях нами были изучены вопросы применения компьютерных технологий в процессе обучения общетехнических дисциплин $[1,2,3,4,6]$.

В процессе обучения курса Общей физики широко применяется форма обучения лабораторный практикум. Студенты снимают показания разных приборов, составляют таблицу наблюдений, строят диаграммы, делают вычисления и заключения. Электронные таблицы удобное средство, с помощью которого можно выполнять самые разнообразные вычисления, легко решать современные инженерные задачи. При работе с таблицами становятся очевидны их характерные особенности, присущие компьютерным программ, как рабочему инструменту. Интересно и эффективно, когда некоторые физические и инженерные (смежные) задачи студенты решают во время обучения курса „Информационные технологий в морском деле“. Именно под таким названием введен курс обучения информационных технологий в БГМА. Многообразие междисциплинарных связей подчеркивает значение информационных технологий в формировании у студентов-моряков современной картины мира. Внедрение такого подхода к обучению производится на основе дополнительных специальных профессиональных компетенций, студенты с интересом закрепляют материал, который им придется еще раз вспоминать при изучении спецдисциплин, определяющих их профессию механика, электромеханика или же штурмана [11].

Постановка задачи. Известный факт, что причиной сопротивления проводимости металла является тепловое движение ионов. Так как скорость дрейфа определяет величину силу тока $(I=q n v S)$, по мере увеличения температуры сила тока в проводнике уменьшается. Это означает, что с ростом температуры сопротивление проводника увеличивается $\left(I=\frac{U}{R}\right)$. Так как электрическое сопротивление металла зависит от температуры, температуру можно вычислить на основе измерений сопротивления металла. Терморезисторы относятся к параметрическим датчикам температуры, поскольку их активное сопротивление зависит от температуры. В нем используется металлическая проволока или пластина. Металлические терморезисторы изготовляют из чистых металлов: меди, платины, никеля, железа, реже из молибдена и вольфрама. При увеличении температуры на $1^{\circ} \mathrm{C}$ сопротивление металлического терморезистора увеличивается на $0,4-0,65 \%$.

Зависимость сопротивление металла от температуры нелинейная, но при небольшом интервале температуры его можно считать линейным и тогда уравнение, связывающее зависимость температуры от сопротивления, имеет вид ([5], 172-173):

$$
\frac{R_{t}-R_{0}}{R_{0}}=1+\alpha t \quad \Rightarrow \quad R_{t}=R_{0}(1+\alpha t)
$$

Или по-другому (в форме линейной регресси):

$$
R_{t}=R_{0}+R_{0} \alpha t
$$

где $R_{t}$ - сопротивление при измеряемой температуре $t, R_{0}-$ сопротивление при температуре $0^{\circ} \mathrm{C}$ (известная величина, одна из спецификаций терморезистора), $\alpha$ - линейный температурный коэффициент (также известная величина, одна из спецификаций терморезистора). Температурный коэффициент физическая характеристика металла, указывающая, как изменяется его электрическое сопротивление с изменением температуры. Именно это свойство металла лежит в основе принципа действия терморезистора. Для платины (часто используется в терморезисторах) значение коэффициента $\alpha=0,00385^{\circ} \mathrm{C}^{-1}$. Однако, в более ранних терморезисторах использовалась другая марка платины, и для них значение $\alpha=0,003902{ }^{\circ} \mathrm{C}^{-1}$. В лабораторных терморезисторах используется очень чистая платина, для которой $\alpha=0,003923^{\circ} \mathrm{C}^{-1}$. Если при вычислении температуры по показаниям терморезистора используется неправильное или неточное значение $\alpha$, то полученные значения температур будут отличаться от истинных.

Задача. В судовой печи случайно был установлен лабораторный терморезистор. В компьютерную систему, управляющую температурой в печи, введено значение $\alpha=0,00385^{\circ} \mathrm{C}^{-1}$. Чему равна истинная температура (вычисленная с коэффициентом $\alpha$, равным $0,003923{ }^{\circ} \mathrm{C}^{-1}$ ), если 
согласно показаниям системы контроля, использующей значение $\alpha=0,00385^{\circ} \mathrm{C}^{-1}$, температура равняется $100^{\circ} \mathrm{C}$ ?

Решение задачи. Мы использовали Arduino для измерения и обработки показаний термистора, после чего преобразовали эти показания. На рисунке 1 приведены две данные а) и b), описывающие зависимость сопротивления двух разных терморезисторов от температуры [5].

\begin{tabular}{|c|c|}
\hline temperature $\left({ }^{\circ} \mathrm{C}\right)$ & resistance (Om) \\
\hline 0 & 100,00 \\
\hline 10 & 103,90 \\
\hline 20 & 107,79 \\
\hline 30 & 111,67 \\
\hline 40 & 115,54 \\
\hline 50 & 119,40 \\
\hline 60 & 123,24 \\
\hline 70 & 127,07 \\
\hline 80 & 130,89 \\
\hline 90 & 134,70 \\
\hline 100 & 138,50 \\
\hline
\end{tabular}

\begin{tabular}{|c|c|}
\hline temperature $\left({ }^{\circ} \mathrm{C}\right)$ & resistance $(\mathrm{Om})$ \\
\hline 0 & 100,00 \\
\hline 10 & 103,90 \\
\hline 20 & 107,80 \\
\hline 30 & 111,70 \\
\hline 40 & 115,60 \\
\hline 50 & 119,50 \\
\hline 60 & 123,40 \\
\hline 70 & 127,30 \\
\hline 80 & 131,20 \\
\hline 90 & 135,10 \\
\hline 100 & 139,00 \\
\hline
\end{tabular}

Pис. 1 (а, б). Исходные данные

Для данных a) и b) рисунка 1 необходимо отдельно проделать следующие шаги:

1) построить в Excel точечную диаграмму (Scatter with only Markers);

2) в диалоговом окне Format Trendline (Рис. 2) на вкладке „,Trendline Options“ из группы „Trend/Regresion Type“, выбрать линейный параметр (linear), установить флажки в строках (Display Equation on chart) - показать уравнение на диаграмме и Display R-squaerd value on chart - поместить значение надежности величины аппроксимации $R^{2}$ на диаграмме); прямой;

3) определить линейный температурный коэффициент с помощью аппроксимирующей

4) определить какой из этих терморезисторов является лабораторным?

Результаты решения в Excel, для зависимостей а) и b) рисунка 1, показаны, соответственно, на рисунках 3 и 4. По величине $R^{2}$ - коэффициента корреляции можно судить о правомерности использования уравнения линейной регрессии. Если его значение находится в диапазоне от 0,9 до 1, то это соотношение можно использовать для анализа результатов. Чем ближе коэффициент корреляции к единице, тем достовернее надежность используемой модели.

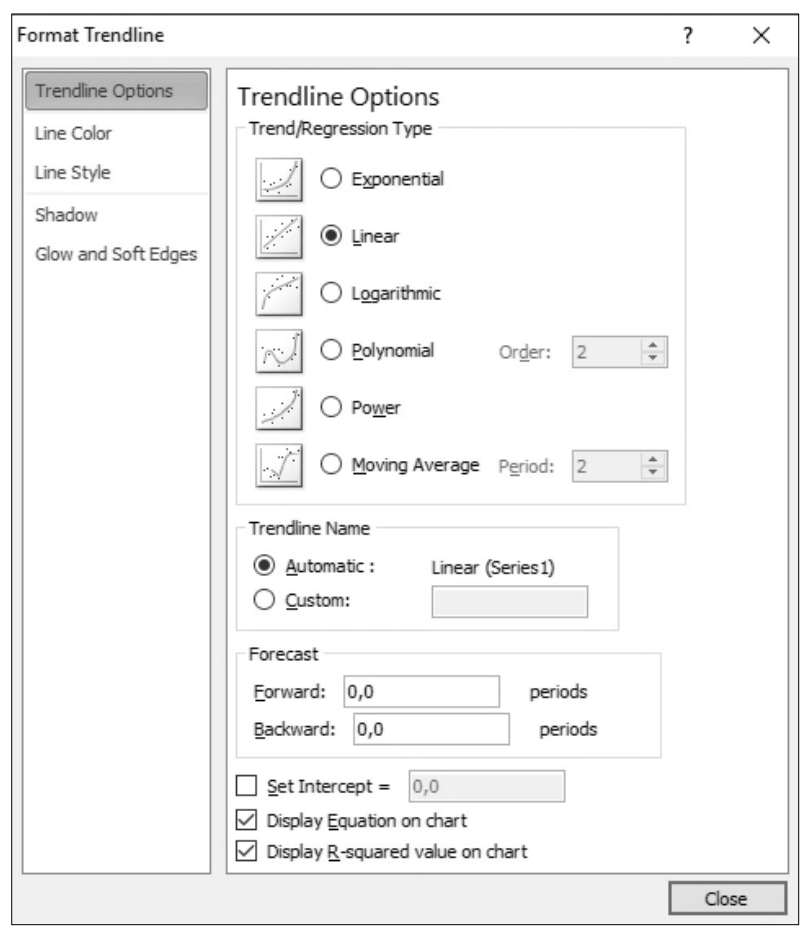

Pис. 2. Диалоговое окно Format Trendline 
Как видно из рисунков 3 и 4 квадрат коэффициента корреляции равен 1, что утверждает то, что действительно построена линейная модель, следовательно, линейная модель может быть использована для анализа результатов. При помощи этой модели можно определить величину линейного температурного коэффициента $\alpha$ и установить, является ли терморегулятор лабораторным.

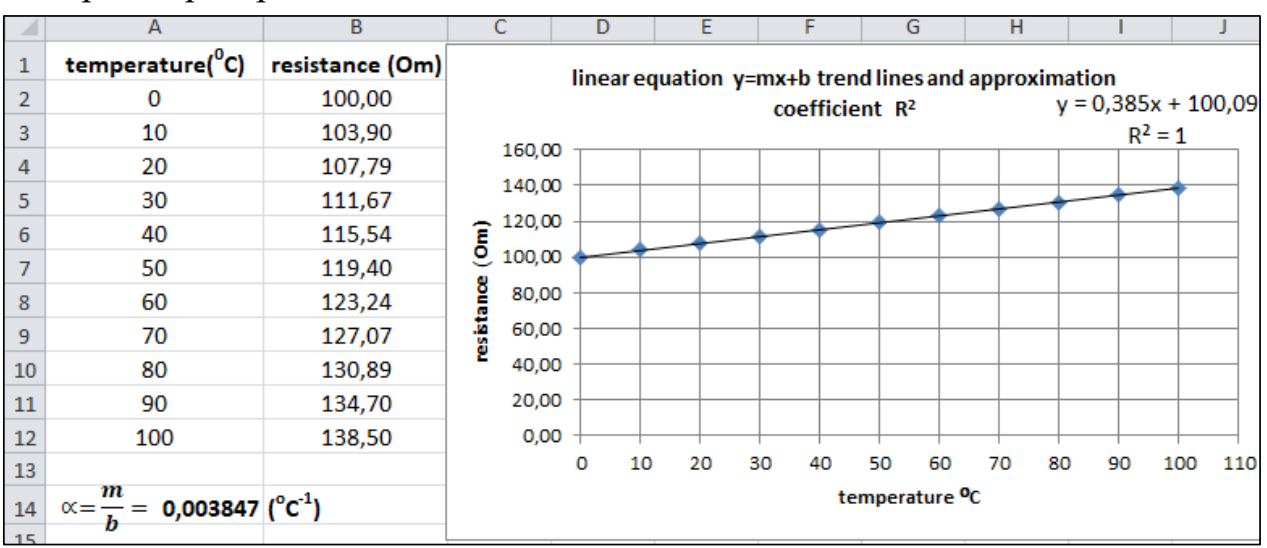

Рис. 3. График линии тренда-линейная модель

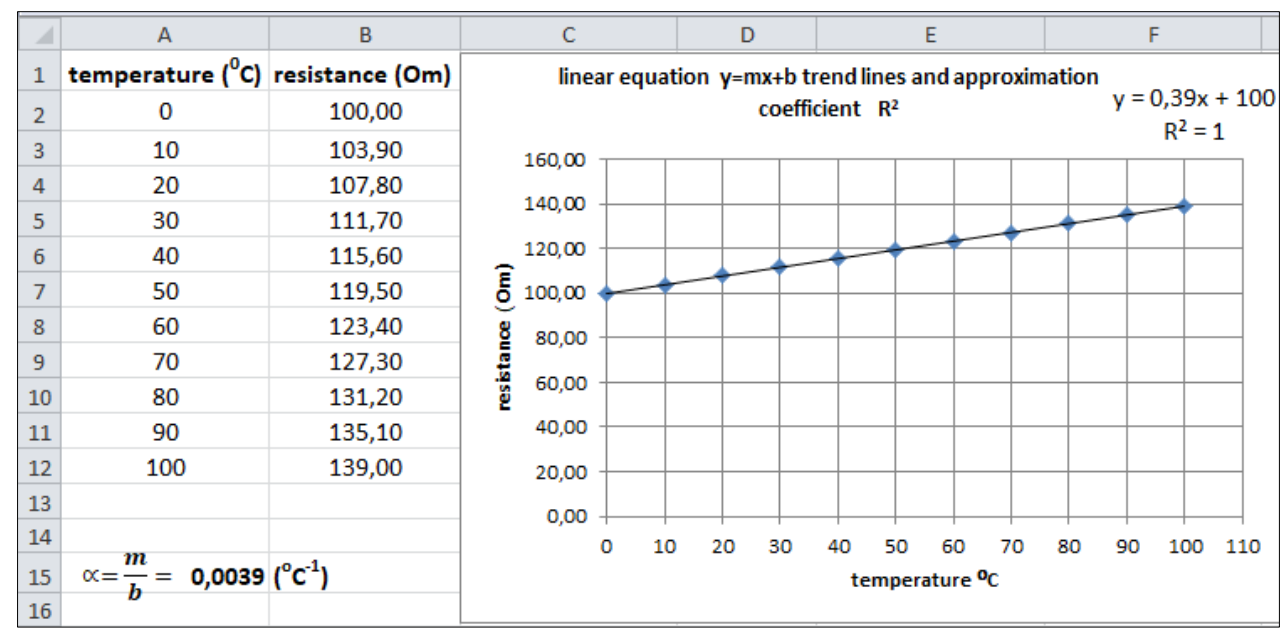

Рис. 4. График линии тренда -линейная модель

Из рисунка 3 видно, что найдены неизвестные $m=0,385$ и $b=100,09$ коэффициенты уравнения $y=m x+b$. Сопоставляя полученные результаты с уравнением зависимости: $R_{t}=$ $R_{0}+\left(R_{0} \alpha\right) t$, записанного в форме линейной регрессии, видно, что $R_{0} \alpha=m$, и $R_{0}=b$. Тогда величина линейного температурного коэффициента определяется путем соотношения: $\alpha=$ $\frac{m}{b}=0,003847\left({ }^{\circ} \mathrm{C}^{-1}\right) \approx 0,00385\left({ }^{\circ} \mathrm{C}^{-1}\right)$. Поскольку в лабораторных термисторах используется очень чистая платина, для которой значение коэффициента $\alpha$ составляет $0,003923{ }^{\circ} \mathrm{C}^{-1} \approx$ $0,0039^{\circ} \mathrm{C}^{-1}$, мы можем заключить, что этот термистор не является лабораторным.

Из рисунка 4 видно, что найдены неизвестные $m=0,39$ и $b=100$ коэффициенты уравнения $y=m x+b$. Аналогичным путем, вычисляем величину линейного температурного коэффициента $\alpha=\frac{m}{b}=0,0039\left({ }^{\circ} \mathrm{C}^{-1}\right)$. Сравнивая полученное значение со значением коэффициента $\alpha=0,003923\left({ }^{\circ} \mathrm{C}^{-1}\right) \approx 0,0039\left({ }^{\circ} \mathrm{C}^{-1}\right)$, мы можем заключить, что этот термистор действительно является лабораторным.

Результаты и выводы. Выполненная нами работа не претендует на исчерпывающее решение проблемы, касающейся возможностей интеграции общетехнических дисциплин. Но применение таких пересечений между смежными дисциплинами в учебном процессу способствует повышению качества высшего образования, что в полной мере способствует формированию нового поколения новыми компетенциями.

На основе наших наблюдений можно утверждать, что использование в учебном процессе меж предметной связи обеспечивает достаточно высокий уровень усвоения знаний, способствует динамике развития умений и навыков выпускников с техническим направлением 
подготовки, способствуя при этом формированию их интегративных компетенций. По итогам наших исследований можно сделать вывод, что междисциплинарная связь позволят формированию интегративных компетенций студентов. Перспективными проблемами являются: разработка новых видов самостоятельной работы для студентов, а также возможность оптимизации усвоения смежных дисциплин.

Аналогичным методом можно решить следующие практические задачи: 1) "манометр" - вычисление давления; 2) “основные характеристики потока жидкости”; 3) “гидростатика" давление на дно сосуда; 4) “уравнение идеального газа"; 5) “блоки” - определить: а) натяжение нити; б) ускорение груза; в) сила, приложенная к концу троса лебедки; 6) “движение тела, брошенного под углом”; 7) “диаграмма натяжений” и др. [5].

Заключение. Научная новизна исследования заключается в определении факторов педагогической целесообразности реализации возможностей средств компьютерных технологий в процессе обучения смежных общетехнических дисциплин. Подведя итоги, можно сделать вывод о целесообразности применения междисциплинарных принципов в обучении студентов в Морских Учебных заведений, подтверждённый педагогическим опытом [7, 8, 9], [10]. Применение этого метода будет способствовать совершенствованию учебного процесса, усвоению сложного материала, установить связь между студентами и формами обучения. По сравнению с традиционным подходом, предлагаемый подход позволяет решать задачи дальнейшего развития и углубления методов обучения и воспитания студентов - будущих моряков, тем самым повысить мотивацию обучения, достичь успехов в интеллектуальном и профессиональном развитии. В итоге наш выпускник будет силен тем, что он научится не запоминать, а размышлять, думать, доказывать, сомневаться, преодолевать. Тогда он и становится ученым, или настоящим профессионалом и специалистом своего дела.

\section{ЛИТЕРАТУРА}

1. Телия Т.Н. Расчет оптимальных параметров кривошипно-шатунного механизма в Excell, International Academy of Sceince and Higher Education London, United Kingdom, Global International Scientific Analytical project, Published by IASHE, LXXXVIII International Research and Practice Conference and II stage of the Championship in Technical science, Architecture and Construction, London, August 28-September 02, 2014, pp. 73-75;

2. Телия Т.Н. Методика оценки прогноза результатов в области MS Excel, International Academy of Sceince and Higher Education London, United Kingdom, Global International Scientific Analytical project, LXXXVI International Research and Practice Conference and II stage of the Championship in Economics and Management, Jurisprudence, Sociological, Political and Military sciences, London, July 24-29, 2014, pp. 39-41;

3. Телия Т.Н, Оптимизация штатного расписания морской компаний, International Academy of Sceince and Higher Education London, United Kingdom, Global International Scientific Analytical project, CVII International Research and Practice Conference and II stage of the Championship in Economics, Management and Jurisprudence, Sociology, Political and Military sciences, London, August 26-31, 2015, pp. 35-37;

4. Telia T.N., Motskobili I.R. On the solution of one linear optimization objectives in /O решении одной линейной оптимизационной задачи в Excel, Problems of the Development of Modern Science: theory and practice, Cartero Publishing House, Spain 2018, pp. 29-32;

5. Ronald W. Larsen. Engineering With Excel. Pearson Education, USA, 2017;

6. M.Diasamidze, I.Motskobili, Ts. Gvinjilia, Takidze I. Evaluation of the "Quality of Education" in higher educational institutions (on the example of the Batumi State Maritime Academy). "Оценка «качества образования» в высших учебных заведениях (на примере Батумской государственной морской академии)" Scientific Journal Fundamental and applied researches in practice of leading scientific schools, V.31, $\# 1,2019$, pp. 54-57;

7. M. Diasamidze, I. Motskobili, Ts. Gvindzhiliya, I. Takidze, Implementation of the conceptual model of forecasting the needs of qualified specialists in the Region, "Реализация концептуальной модели прогнозирования потребностей в квалифицированных специалистах в регионе” Baku 2018;

8. M.Diasamidze, I.Motscobili, I.Takidze, Ts.Gvinjilia, Realization of the conceptualization of technical education Specialists in the region, 4 ways of solving them", "Реализация концептуализации технического образования специалистов региона, 4 пути их решения », Jun3 24, 2018, Batumi-Georgia;

9. M.Diasamidze, Gravity and Maritime Navigation "Гравитация и морская навигация" (IX International Conference of the Georgian Mathematical Association, September 3-8, 2018, Batumi-Tbilisi;

10. M.Diasamidze, I.Takidze, Ts.Gvinjilia. Educational institutions of higher education in the process of teaching methods (the example of Batumi State Maritime Academy). HENTI 2017, The Second International Conference: Higher Education-new Technologies and Innovations, Kutaisi, May 1-2, 2017;

11. M. Diasamidze, I. Takidze, C. Gvindzhiliya, Natural Sciences in the Service of Improving Maritime Transport, “Естественные науки на службе совершенствования морского транспорта," Baku, 2017. 